\title{
EFEITO DE TREINAMENTO EM MINI-TRAMPOLIM EM SOLO E EM ÁGUA: BENEFÍCIOS ANTROPOMÉTRICOS, ATLÉTICOS E DE QUALIDADE DE VIDA.
}

\section{Effects of water and ground rebound exercise programmes on young women quality of life and anthropometric and athletic correlates}

\author{
Paula Tatiane Alonso ${ }^{1}$; Tatiana Coletto dos Anjos ${ }^{1}$; Juliana Paula Leite ${ }^{1}$; Aguinaldo Gonçalves ${ }^{1}$; Carlos Roberto Padovani ${ }^{2}$ \\ 1Faculdade de Educação Física/UNICAMP/Campinas/SP; ' Instituto de Biociências/ UNESP/ Botucatu/SP
}

\begin{abstract}
Resumo: O objetivo deste estudo foi explorar o efeito de programa no mini-trampolim em solo e na água sobre variáveis antropométricas (peso; índice de massa corpórea; circunferência de cintura, quadril e relação entre estas; média dos valores das dobras cutâneas supra-ilíaca, subescapular e da coxa; percentual de massa gorda e magra), atléticas (salto com e sem auxílio dos membros superiores, flexibilidade, consumo e absorção de oxigênio, freqüência cardíaca logo após o repouso, resistência muscular localizada de membros inferiores, superiores e abdominais) e os domínios de qualidade de vida. Os exercícios eram coreografados e realizados durante 16 semanas de duração, três vezes semanais, com 45 minutos. Foram avaliadas, nos momentos pré e pós intervenção, 46 mulheres jovens, sedentárias, entre 19 e 35 anos de idade (média de 23,97士4,06), divididas em dois grupos. Aplicou-se a técnica da análise de variância não-paramétrica para medidas repetidas em grupos com dois fatores, sendo um deles dependente. A associação entre o conjunto de variáveis, em cada grupo, foi investigada pela técnica multivariada de interdependência, apresentada pela função e carga canônica. Observou-se significância estatística (5\%) nas relações entre ambos os grupos de variáveis com os domínios de qualidade de vida físico ( $p<0,05)$; psicológico $(p<0,0001)$; social $(p<0,01)$ e ambiental $(p<0,005)$ na modalidade aquática, enquanto que, na terrestre, o mesmo não ocorreu. No entanto, na impossibilidade de adotar-se a primeira alternativa, a segunda não deve ser desconsiderada, pois aí também diferenças significativas foram registradas na comparação antes e após intervenção nos valores individuais das variáveis.
\end{abstract}

Palavras-chave: Indicadores antropométricos; variáveis atléticas; qualidade de vida; mini-trampolim; treinamento.

Abstract: The purpose of this study is to explore effects of a ground and water rebound exercise programme on quality of life domains (QOLD) and anthropometric and athletic correlates. Weight, body mass index, waist circunference, hip circunference, ratio between these two measures, skinfolds thickness measurement, fat percentage, flexibility, uptake and consumption of oxygen, after rest cardiac frequency, located muscular strenght of lower and upper members and QOLD were investigated. Low - impact aerobic choreographic exercises were performed during sixteen weeks on a three weekly sessions of 45 minutes basis. Pre and post period assessments were carried out on 45 young sedentary women, aged between 19 and 35 years old (average 23, 97+4,06). Analysis of variance for repeated measures in two groups was applied. Association between all variables in each group was searched by interdependence multivariate technique, featured by canonic load. Relations between athletic and anthropometric variables revealed statistical significance on all QOLDs (physical, $p<0,05$; psychological, $p<0,0001$; social, $p<0,01$ and environmental, $p<0,005$ ) in water and on none of them in ground rebound exercise programmes. Even when there is no condition of developing the first one, the second should be considered, because significative differences were observed in comparing values before and after such intervention on studied variables.

Key words: Anthropometric measures, athletic correlates, quality of life, rebound exercise, training

Aceito em 11/02/2009 - Rev. Educ. Fís. 2009 Jun: 145: 3-10. Rio de Janeiro - RJ - Brasil

\section{INTRODUÇÃO}

O estilo de vida sedentário é considerado hoje o principal causador de doenças crônicas como as arteriopatias coronarianas, diabetes, hipertensão, obesidade, além de respectivos fatores associados: redução acentuada e progressiva de massa muscular e força, flexibilidade e equilíbrio(1,2). Benefícios preventivos nesse sentido são reconhecidos pela ação dos exercícios físicos ${ }^{(3,4)}$. De fato, a literatura tem relatado que auxiliam no desenvolvimento da aptidão física básica, 
resistência ao esforço, agilidade, coordenação, força muscular(5). Mais amplamente autores como Oliveira Filho e Shiromoto(6) demonstram, que, realizados de forma regular, pelo menos três vezes por semana, agem positivamente sobre aspectos relacionados à qualidade de vida dos indivíduos, levando-os a níveis mais próximos dos padrões desejáveis em termos de saúde.

Dentre os aparelhos disponíveis no mercado, tem-se podido contar, mais recentemente, com o mini-trampolim. Pauta-se pelo desenvolvimento de práticas aeróbias e de resistência muscular, com intensidade, duração e freqüência préestabelecidas, priorizando a musculatura dos membros inferiores (quadríceps, glúteos) e abdome. $O$ instrumento permite a realização de exercícios que envolvem força da gravidade, aceleração e desaceleração, devido à superfície elástica e sistema de fixação de molas de resistência especial ${ }^{(7)}$.

Relacionando-se estas duas ordens de fatos, objetivou-se determinar possíveis benefícios sobre variáveis antropométricas, atléticas e de qualidade de vida pelo minitrampolim como equipamento de treinamento. Em termos específicos, objetivouse, também no presente estudo, comparar tais aspectos de maneira intra e inter grupos, segundo sua utilização em solo e em água, referenciandoos à questão da Qualidade de Vida.

\section{METODOLOGIA}

Iniciou-se com a participação de 51 mulheres jovens que se voluntariaram para o projeto anunciado através de procedimentos correntes das atividades de Extensão da Universidade. Como critérios de inclusão, deviam ser sedentárias (três meses afastadas de atividades físicas regulares), saber nadar (pois a altura da água, durante a execução, deveria se localizar no processo xifóide) e apresentar atestado médico indicando aptidão para atividades físicas leves e moderadas. Adotou-se para exclusão inadequação das disponibilidades pessoais ao horário oferecido. Alocaram-se 22 e 24 participantes em cada um dos grupos formados para treinamento em solo e água, segundo decisão pessoal. Adoção deste cuidado ético mandatório permitiu casualização parcial, configurando o delineamento como quaseexperimental(8). Contou-se, ao longo do processo, com cinco perdas, potencialmente já previstas, decorrentes do fato de que grande parcela era formada por estudantes da universidade, e os períodos de compromissos acadêmicos acabaram influenciando na ausência.

Possíveis benefícios e testes foram previamente esclarecidos e a partir daí optaram por assinar livremente o consentimento formal, aprovado pelo Comitê de Ética em Pesquisa da Universidade Estadual de Campinas- UNICAMP, (Faculdade de Ciências Médicas), parecer projeto número 324/2004.

Os protocolos de avaliação, com horários (mesmo período do dia) e avaliadores fixos, foram aplicados no Laboratório de Antropologia Física em cooperação com o Laboratório de Atividade Física e Performance Humana, ambos na Faculdade de Educação Física (FEF) da UNICAMP, em diferentes sessões, de forma que um procedimento não interferisse no outro.

Obtiveram-se valores das variáveis:

1) antropométricas: a) relação entre circunferências da cintura pélvica e quadril, por meio de fita metálica flexível, de acordo com as técnicas convencionais, descritas por Heyward e Stolarczyz ${ }^{(9)} ;$ b) índice de massa corporal (IMC) e demais medidas decorrentes de acordo com os procedimentos descritos por Gordon et al. (10). Especificamente o percentual de massa gorda aí mencionado e aplicado no presente projeto é obtido pelo emprego da fórmula de Siri, baseada na densidade corporal (DC), em que: 
$D C=1.099421-0,0009929(X 1)+0,0000023$

$$
\begin{aligned}
& (X 1) 2-0,0001392(X 2) \\
& \% G C=[495 / D C]-450
\end{aligned}
$$

onde, X1 é a somatória das médias das dobras cutâneas triciptal, suprailíaca e medial da coxa e X2, a idade do indivíduo em anos; c) dobras cutâneas das regiões supra-ilíaca, subescapular e coxa direita, com adipômetro calibrado Lange $\AA$, capacidade para 50mm, pressão constante de 9,3 $\mathrm{g} / \mathrm{mm}^{2}$ na superfície de contato e precisão de 0,5 $\mathrm{mm}^{(11)}$;

2) atléticas: a) força de membros inferiores com e sem auxílio de membros superiores, no "Sargent Test"; b) flexibilidade no Banco de Wells, avaliando região posterior das pernas (tendões e musculatura poplítea), quadris e coluna lombar, no teste de sentar e alcançar seguindo o protocolo proposto pelo $\mathrm{ACSM}^{(5)}$; c) resistência muscular localizada de membros inferiores, através de agachamentos e resistência abdominal; d) freqüência cardíaca máxima em teste e consumo de oxigênio (VO2), limitadamente, pelo protocolo do Banco de Astrand, com banco de $33 \mathrm{~cm}$ de altura para mulheres, no qual a pessoa deveria subir e descer em ritmo de 22,5 passadas por minuto durante período de 5 minutos;

3) referentes a qualidade de vida pelo WHOQOLBref, questionário composto por 26 questões de linguagem simples e perguntas curtas, padronizado e validado pela Organização Mundial da Saúde, para uso tanto a nível nacional quanto internacional, investigando quatro domínios do quotidiano: físico, psicológico, relações sociais e meio ambiente ${ }^{(12)}$.

O programa de treinamento ocorreu em 16 semanas, com três aulas semanais, de 45 minutos de duração. As sessões, iniciadas logo após os primeiros testes, foram realizadas nas dependências atléticas da FEF, em dois horários diferentes. Adotaram-se rotinas compostas por pré-coreografias ("mix") controladas pelas batidas musicais por minuto (bpm), com intensidade crescente a cada "mix": no primeiro, com média de 136 bpm, no segundo, de 140 e no terceiro e último, de 142. Cada "mix" continha onze músicas pré-coreografadas, a primeira destinada ao aquecimento dos membros superiores e inferiores, e as nove consecutivas, para movimentos específicos da modalidade. A última constituía-se de exercícios de alongamentos e relaxamentos.

As variáveis antropométricas, atléticas e qualidade de vida nos dois grupos avaliados nos momentos pré e pós intervenção, foram consideradas pela técnica da análise de variância para o modelo de medidas repetidas em grupos independentes, a dois fatores, sendo um dependente(13). A associação entre o conjunto de variáveis antropométricas, atléticas e qualidade de vida, em cada grupo, foi investigada por meio da técnica multivariada de interdependência da correlação canônica, apresentada pela função e carga canônica ${ }^{(14)}$. Todas as discussões foram realizadas no nível de $5 \%$ de significância, segundo Gonçalves ${ }^{(15)}$.

\section{RESULTADOS}

Como valores de centralidade e dispersão da idade das pessoas estudadas, obteve-se média e desvio padrão de 23,97 $\pm 4,06$.

As TABELAS 1 e 2 apresentam os valores de média e desvio padrão das variáveis referentes a antropometria e composição corporal analisadas nos períodos pré e pós-treinamento e a TABELA 3 , os resultados dos domínios de avaliação da percepção subjetiva de qualidade de vida. As TABELAS 4 e 5 registram os coeficientes de associação entre variáveis antropométricas, atléticas e qualidade de vida, estatisticamente significantes entre as praticantes da modalidade na água e não significantes ( $p>0,05)$, os revelados pelas usuárias do mini-trampolim no solo. 
TABELA 1

MÉDIA, DESVIO-PADRÃO E COMPARAÇÃO ESTATIISTICA DAS VARIÁVEIS ANTROPOMÉTRICAS, SEGUNDO MOMENTO E GRUPO DE AVALIAÇÃO

\begin{tabular}{|c|c|c|c|c|}
\hline \multirow{2}{*}{ VARIÁVEL } & & \multicolumn{2}{|c|}{ MOMENTOS } & \multirow{2}{*}{$\begin{array}{l}\text { VALOR } \\
\text { DE p }\end{array}$} \\
\hline & & INICIAL & FINAL & \\
\hline $\begin{array}{c}\text { Massa Corpórea } \\
(\mathbf{k g})\end{array}$ & $\begin{array}{l}\text { Na água } \\
\text { No solo }\end{array}$ & $\begin{array}{l}55,84 \pm 8,50 \\
56,80 \pm 6,50\end{array}$ & $\begin{array}{l}55,42 \pm 8,56 \\
56,48 \pm 6,08\end{array}$ & $\begin{array}{l}p>0,05 \\
p>0,05\end{array}$ \\
\hline $\begin{array}{c}\text { Índice de Massa } \\
\text { Corpórea } \\
\text { (kg/cm2) }\end{array}$ & $\begin{array}{l}\text { Na água } \\
\text { No solo }\end{array}$ & $\begin{array}{l}21,63 \pm 2,67 \\
21,69 \pm 2,87\end{array}$ & $\begin{array}{l}21,46 \pm 2,62 \\
21,57 \pm 2,68\end{array}$ & $\begin{array}{l}p>0,05 \\
p>0,05\end{array}$ \\
\hline $\begin{array}{c}\text { Relação Cintural } \\
\text { Quadril }\end{array}$ & $\begin{array}{l}\text { Na água } \\
\text { No solo }\end{array}$ & $\begin{array}{l}0,7432 \pm 0,0398 \\
0,7133 \pm 0,0540\end{array}$ & $\begin{array}{l}0,7304 \pm 0,0329 \\
0,7273 \pm 0,0625\end{array}$ & $\begin{array}{l}{ }^{*} p<0,05 \\
p<0,05\end{array}$ \\
\hline $\begin{array}{c}\text { Média da Dobra } \\
\text { Subescapular } \\
(\mathrm{mm})\end{array}$ & $\begin{array}{l}\text { Na água } \\
\text { No solo }\end{array}$ & $\begin{array}{l}21,73 \pm 7,25 \\
20,55 \pm 6,75\end{array}$ & $\begin{array}{l}22,70 \pm 7,19 \\
21,39 \pm 7,96\end{array}$ & $\begin{array}{l}p>0,05 \\
p>0,05\end{array}$ \\
\hline $\begin{array}{c}\text { Média da Dobra } \\
\text { Supra-ilíaca } \\
(\mathrm{mm})\end{array}$ & $\begin{array}{l}\text { Na água } \\
\text { No solo }\end{array}$ & $\begin{array}{l}25,90 \pm 7,03 \\
27,77 \pm 8,20\end{array}$ & $\begin{array}{l}28,18 \pm 9,20 \\
26,77 \pm 9,25\end{array}$ & $\begin{array}{l}{ }^{*} p<0,05 \\
p>0,05\end{array}$ \\
\hline $\begin{array}{c}\text { Média da Dobra da } \\
\text { Coxa } \\
(\mathrm{mm})\end{array}$ & $\begin{array}{l}\text { Na água } \\
\text { No solo }\end{array}$ & $\begin{array}{l}38,95 \pm 6,04 \\
40,97 \pm 7,46\end{array}$ & $\begin{array}{c}38,45 \pm 8,89 \\
43,05 \pm 10,51\end{array}$ & $\begin{array}{l}p>0,05 \\
p>0,05\end{array}$ \\
\hline $\begin{array}{c}\text { Massa Gorda } \\
(\%)\end{array}$ & $\begin{array}{l}\text { Na água } \\
\text { No solo }\end{array}$ & $\begin{array}{l}30,44 \pm 5,16 \\
30,94 \pm 4,83\end{array}$ & $\begin{array}{l}31,07 \pm 6,16 \\
31,27 \pm 5,91\end{array}$ & $\begin{array}{l}p>0,05 \\
p>0,05\end{array}$ \\
\hline $\begin{array}{c}\text { Massa Magra } \\
(\%)\end{array}$ & $\begin{array}{l}\text { Na água } \\
\text { No solo }\end{array}$ & $\begin{array}{l}69,56 \pm 5,16 \\
69,06 \pm 4,83\end{array}$ & $\begin{array}{l}68,93 \pm 6,16 \\
68,73 \pm 5,91\end{array}$ & $\begin{array}{l}p>0,05 \\
p>0,05\end{array}$ \\
\hline
\end{tabular}

* Apresenta significância estatística.

TABELA 2

MÉDIA, DESVIO-PADRÃO E COMPARAÇÃO

ESTATÍSTICA DAS VARIÁVEIS ATLÉTICAS, SEGUNDO MOMENTO E GRUPO DE AVALIAÇÃO

\begin{tabular}{|c|c|c|c|c|}
\hline \multirow{2}{*}{\multicolumn{2}{|c|}{ VARIÁVEL }} & \multicolumn{2}{|c|}{ MOMENTOS } & \multirow{2}{*}{$\begin{array}{l}\text { VALOR } \\
\text { DE p }\end{array}$} \\
\hline & & INICIAL & FINAL & \\
\hline \multicolumn{5}{|c|}{ Força de Membros } \\
\hline $\begin{array}{l}\text { Salto sem auxílio } \\
(\mathrm{m})\end{array}$ & $\begin{array}{l}\text { Na água } \\
\text { No solo }\end{array}$ & $\begin{array}{l}0,150 \pm 0,046 \\
0,167 \pm 0,085\end{array}$ & $\begin{array}{l}0,268 \pm 0,039 \\
0,310 \pm 0,113\end{array}$ & $\begin{array}{l}{ }^{*} p<0,01 \\
{ }^{*} p<0,01\end{array}$ \\
\hline $\begin{array}{l}\text { Salto com auxílio } \\
(\mathrm{m})\end{array}$ & $\begin{array}{l}\text { Na água } \\
\text { No solo }\end{array}$ & $\begin{array}{l}0,199 \pm 0,053 \\
0,207 \pm 0,093\end{array}$ & $\begin{array}{l}0,307 \pm 0,044 \\
0,356 \pm 0,112\end{array}$ & $\begin{array}{l}{ }^{*} p<0,01 \\
p>0,05\end{array}$ \\
\hline Flexibilidade $(\mathrm{cm})$ & $\begin{array}{l}\text { Na água } \\
\text { No solo }\end{array}$ & $\begin{array}{l}25,70 \pm 11,30 \\
28,17 \pm 08,21\end{array}$ & $\begin{array}{l}29,55 \pm 09,64 \\
33,08 \pm 07,78\end{array}$ & $\begin{array}{l}{ }^{*} p<0,05 \\
p>0,05\end{array}$ \\
\hline $\begin{array}{c}\text { Freqüência } \\
\text { cardíaca de } \\
\text { recuperação (bpm) }\end{array}$ & $\begin{array}{l}\text { Na água } \\
\text { No solo }\end{array}$ & $\begin{array}{l}174,80 \pm 15,26 \\
172,00 \pm 22,97\end{array}$ & $\begin{array}{l}161,55 \pm 21,18 \\
156,20 \pm 18,25\end{array}$ & $\begin{array}{l}{ }^{*} p<0,05 \\
{ }^{*} p<0,05\end{array}$ \\
\hline $\mathrm{VO}^{2}$ máximo (I) & $\begin{array}{l}\text { Na água } \\
\text { No solo }\end{array}$ & $\begin{array}{l}1,84 \pm 0,43 \\
1,90 \pm 0,46\end{array}$ & $\begin{array}{l}2,01 \pm 0,50 \\
2,32 \pm 0,60\end{array}$ & $\begin{array}{l}{ }^{*} p<0,05 \\
{ }^{*} p<0,01\end{array}$ \\
\hline \multicolumn{5}{|c|}{ Resistência Muscular Localizada } \\
\hline $\begin{array}{l}\text { Abdominal } \\
\text { (repetições) }\end{array}$ & $\begin{array}{l}\text { Na água } \\
\text { No solo }\end{array}$ & $\begin{array}{l}26,70 \pm 5,96 \\
26,38 \pm 6,14\end{array}$ & $\begin{array}{l}28,00 \pm 6,19 \\
29,79 \pm 5,83\end{array}$ & $\begin{array}{l}p>0,05 \\
{ }^{*} p<0,01\end{array}$ \\
\hline $\begin{array}{l}\text { Membros Inferiores } \\
\text { (repetições) }\end{array}$ & $\begin{array}{l}\text { Na água } \\
\text { No solo }\end{array}$ & $\begin{array}{l}28,60 \pm 3,93 \\
31,75 \pm 4,30\end{array}$ & $\begin{array}{l}32,05 \pm 3,65 \\
36,21 \pm 4,02\end{array}$ & $\begin{array}{l}{ }^{*} p<0,01 \\
{ }^{*} p<0,01\end{array}$ \\
\hline $\begin{array}{l}\text { Membros } \\
\text { Superiores } \\
\text { (repetições) }\end{array}$ & $\begin{array}{l}\text { Na água } \\
\text { No solo }\end{array}$ & $\begin{array}{c}22,75 \pm 6,27 \\
---\end{array}$ & $\begin{array}{c}27,45 \pm 6,74 \\
--\end{array}$ & ${ }^{*} p<0,01$ \\
\hline
\end{tabular}

* Apresenta significância estatística.
TABELA 3

MEDIANA E SEMI-AMPLITUDE TOTAL DA PORCENTAGEM DE PONTOS DOS DOMÍNIOS DO WHOQOL-BREF SEGUNDO GRUPO E MOMENTO DE AVALIAÇÃO

\begin{tabular}{|c|c|c|c|c|}
\hline \multirow{2}{*}{\multicolumn{2}{|c|}{ VARIÁVEL }} & \multicolumn{2}{|c|}{ MOMENTOS } & \multirow{3}{*}{$\begin{array}{c}\text { VALOR } \\
\text { DE p } \\
{ }^{*} p>0,05 \\
p>0,05\end{array}$} \\
\hline & & \multirow{2}{*}{$\begin{array}{c}\text { INICIAL } \\
62,50 \pm 26,79 \\
71,43 \pm 28,58\end{array}$} & \multirow{2}{*}{$\begin{array}{c}\text { FINAL } \\
71,43 \pm 25,00 \\
75,00 \pm 23,22\end{array}$} & \\
\hline Domínio Físico & $\begin{array}{l}\text { Na água } \\
\text { No solo }\end{array}$ & & & \\
\hline $\begin{array}{c}\text { Domínio } \\
\text { psicológico }\end{array}$ & $\begin{array}{l}\mathrm{Na} \text { água } \\
\text { No solo }\end{array}$ & $\begin{array}{l}66,67 \pm 25,00 \\
70,83 \pm 29,17\end{array}$ & $\begin{array}{l}75,00 \pm 20,84 \\
75,00 \pm 25,00\end{array}$ & $\begin{array}{l}p>0,05 \\
p>0,05\end{array}$ \\
\hline Domínio social & $\begin{array}{l}\text { Na água } \\
\text { No solo }\end{array}$ & $\begin{array}{l}75,00 \pm 25,00 \\
75,00 \pm 37,50\end{array}$ & $\begin{array}{l}75,00 \pm 50,00 \\
75,00 \pm 25,00\end{array}$ & $\begin{array}{l}p<0,05 \\
p<0,05\end{array}$ \\
\hline $\begin{array}{l}\text { Domínio } \\
\text { ambiental }\end{array}$ & $\begin{array}{l}\text { Na água } \\
\text { No solo }\end{array}$ & $\begin{array}{l}60,94 \pm 21,88 \\
59,38 \pm 29,69\end{array}$ & $\begin{array}{l}60,94 \pm 25,00 \\
68,75 \pm 29,69\end{array}$ & $\begin{array}{l}p>0,05 \\
p>0,05\end{array}$ \\
\hline
\end{tabular}

* Apresenta significância estatística.

TABELA 4

COEFICIENTES DE ASSOCIAÇÃO ENTRE VARIÁVEIS ATLÉTICAS E QUALIDADE DE VIDA NO GRUPO EM ÁGUA

\begin{tabular}{|c|c|c|c|c|}
\hline \multirow{2}{*}{ VARIÁVEL } & \multicolumn{4}{|c|}{ DOMÍNIOS } \\
\hline & Físıco & PSICOLÓGICO & SOCIAL & AMBIENTAL \\
\hline IMC & $-0,472(-0,316)$ & $-0,471(0,099)$ & $-1,242(-0,449)$ & $-1,988(-0,284)$ \\
\hline Cintura/quadril & $0,468(0,223)$ & $-0,151(-0,080)$ & $0,652(0,005)$ & $0,059(-0,346)$ \\
\hline $\begin{array}{c}\text { Média } \\
\text { da dobra } \\
\text { subescapular }\end{array}$ & $-0,469(-0,439)$ & $-0,303(-0,485)$ & $-0,488(-0,543)$ & $0,083(-0,158)$ \\
\hline $\begin{array}{c}\text { Média da } \\
\text { dobra supra- } \\
\text { ilíaca }\end{array}$ & $-0,065(-0,183)$ & $-0,011(-0,246)$ & $-0,386(-0,569)$ & $-0,130(-0,420)$ \\
\hline $\begin{array}{c}\text { Media da } \\
\text { dobra da coxa }\end{array}$ & $0,346(-0,234)$ & $0,467(0,352)$ & $1,209(-0,098)$ & $0,075(-0,127)$ \\
\hline Massa gorda & $2,958(-0,117)$ & $8,012(-0,414)$ & $4,670(-0,256)$ & $23,631(0,099)$ \\
\hline Massa magra & $2,552(-0,066)$ & $7,837(0,556)$ & $4,485(0,319)$ & $23,265(0,239)$ \\
\hline $\begin{array}{l}\text { Salto sem } \\
\text { auxilio }\end{array}$ & $0,544(-0,352)$ & $-0,072(0,491)$ & $0,065(0,377)$ & $-0,222(-0,011)$ \\
\hline $\begin{array}{l}\text { Salto com } \\
\text { auxilio }\end{array}$ & $-0,337(0,501)$ & $0,573(0,651)$ & $-0,155(0,460)$ & $-0,779(-0,052)$ \\
\hline Flexibilidade & $0,741(0,605)$ & $0,355(0,288)$ & $0,043(0,283)$ & $0,469(0,617)$ \\
\hline $\begin{array}{l}\text { Freqüência } \\
\text { cardíaca }\end{array}$ & $-0,100(0,109)$ & $-0,251(0,309)$ & $-0,432(-0,136)$ & $-0,046(0,469)$ \\
\hline $\mathrm{VO}^{2}$ & $0,445(0,290)$ & $0,299(0,469)$ & $0,373(0,378)$ & $0,814(0,041)$ \\
\hline Abdominais & $0,357(0,205)$ & $-0,92(0,105)$ & $0,899(0,114)$ & $0,133(0,355)$ \\
\hline $\begin{array}{l}\text { Membros } \\
\text { inferiores }\end{array}$ & $-0,049(0,106)$ & $-0,029(-0,109)$ & $0,148(-0,041)$ & $0,609(0,164)$ \\
\hline $\begin{array}{l}\text { CORRELAÇÃO } \\
\text { CANÔNICA }\end{array}$ & 0,94 & 0,99 & 0,96 & 0,97 \\
\hline p-VALOR & ${ }^{*} p<0,05$ & ${ }^{*} p<0,0001$ & ${ }^{*} p<0,01$ & ${ }^{*} p<0,005$ \\
\hline
\end{tabular}

*Apresenta significância estatística. 
TABELA 5

COEFICIENTES DE ASSOCIAÇÃO ENTRE

VARIÁVEIS ATLÉTICAS E DOMÍNIOS DE QUALIDADE DE VIDA NO GRUPO EM SOLO

\begin{tabular}{|c|c|c|c|c|}
\hline \multirow{2}{*}{ VARIÁVEL } & \multicolumn{4}{|c|}{ DOMíNIOS } \\
\hline & Físıco & PSICOLÓGICO & SOCIAL & AMBIENTAL \\
\hline IMC & $-0,115(0,321)$ & $-0,233(-0,518)$ & $1.055(0,134)$ & $-0,360(-0,288)$ \\
\hline Cintura/quadril & $-0,292(0,054)$ & $-0,177(-0,214)$ & $-0,389(-0,283)$ & $-0,418(-0,298)$ \\
\hline $\begin{array}{c}\text { Média } \\
\text { da dobra } \\
\text { subescapular }\end{array}$ & $0,783(-0,080)$ & $0,771(0,017)$ & $-0,020(-0,261)$ & $0,503(-0,001)$ \\
\hline $\begin{array}{c}\text { Média da } \\
\text { dobra supra- } \\
\text { ilíaca }\end{array}$ & $-0,372(-0,787)$ & $0,002(-0,172)$ & $-0,187(0,312)$ & $0,187(-0,060)$ \\
\hline $\begin{array}{c}\text { Media da } \\
\text { dobra da coxa }\end{array}$ & $-0,477(-0,633)$ & $-0,561(-0,445)$ & $0,219(-0,169)$ & $-0,317(0,128)$ \\
\hline Massa gorda & $1,123(2.201)$ & $-0,629(-0,003)$ & $1,290(0,007)$ & $-0,090(0,073)$ \\
\hline Massa magra & $-0,279(-1,983)$ & $-0,059(-0,166)$ & $0,726(0,201)$ & $0,305(0,064)$ \\
\hline $\begin{array}{l}\text { Salto sem } \\
\text { auxilio }\end{array}$ & $-0,171(0,247)$ & $0,001(0,662)$ & $0,894(0,235)$ & $-0,309(-0,038)$ \\
\hline $\begin{array}{l}\text { Salto com } \\
\text { auxilio }\end{array}$ & $0,051(0,811)$ & $0,077(0,212)$ & $0,236(0,243)$ & $-0,150(-0,016)$ \\
\hline Flexibilidade & $0,459(1,030)$ & $0,294(0,089)$ & $-0,196(0,279)$ & $0,988(0,664)$ \\
\hline $\begin{array}{l}\text { Freqüência } \\
\text { cardíaca }\end{array}$ & $1,142(-1,205)$ & $-0,595(-0,546)$ & $-2,179(-0,099)$ & $-0,165(-0,312)$ \\
\hline $\mathrm{vo}^{2}$ & $1,824(1,657)$ & $-0,044(0,386)$ & $-1,902(0,065)$ & $-0,206(0,209)$ \\
\hline Abdominais & $-0,245(-1,299)$ & $-0,475(-0,468)$ & $0,995(0,186)$ & $-0,412(-0,055)$ \\
\hline $\begin{array}{l}\text { Membros } \\
\text { inferiores }\end{array}$ & $0,229(-0,334)$ & $-0,021(0,001)$ & $0,48(0,551)$ & $-0,466(-0,248)$ \\
\hline $\begin{array}{l}\text { CORRELAÇÃO } \\
\text { CANÔNICA }\end{array}$ & 0,76 & 0,77 & 0,61 & 0,84 \\
\hline p-VALOR & $p>0,05$ & $p>0,05$ & $p>0,05$ & $p>0,05$ \\
\hline
\end{tabular}

* Apresenta significância estatística.

Com efeito, aí estabelecidos como variáveis dependentes, os domínios (físico, psicológico, social e ambiental) e independentes, as demais (como IMC, resistência de membros inferiores, por exemplo), exploraram-se respectivas associações no interior de cada grupo. Para sua interpretação, os valores colocados antes dos parênteses são os coeficientes da função. Especificamente, no grupo em água, existe associação significativa entre domínio físico e as variáveis atléticas; a pergunta natural que segue à significância é indagar qual (ou quais) variáveis das observadas está mais associada à função canônica atlética? A resposta encontra-se na obtenção da correlação entre as variáveis originais e as canônicas, denominadas de "canonical loadings" ou cargas canônicas, cujos valores encontram-se entre os parênteses. Resulta, assim, que as variáveis associadas com maior intensidade à canônica atlética são flexibilidade, salto com auxílio e média subescapular. Como não se dispõe de possibilidade de apresentação gráfica para correlação canônica, visto que deveria ser feita em espaço acima do tridimensional, representando-se por barras o valor que está dentro dos parênteses ao quadrado, tem-se a FIGURA 1 como correspondência à primeira linha da TABELA 4 , na modalidade aquática.

\section{FIGURA 1}

REPRESENTAÇÃO DA VARIÁVEL ANTROPOMÉTRICA IMC NA RELAÇÃO

CANÔNICA AOS DOMÍNIOS DE QUALIDADE DE VIDA, NA MODALIDADE DE MINI-TRAMPOLIM PRATICADA NA ÁGUA

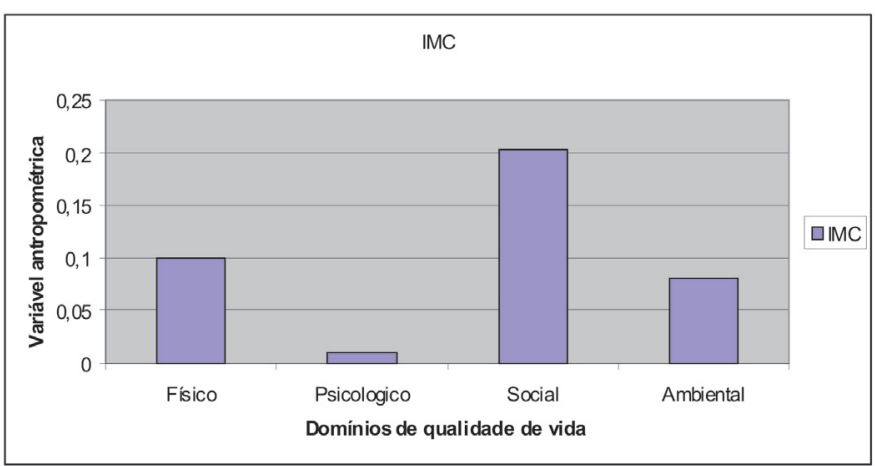

Em relação às variáveis que mais se associam com o domínio,quando o valor dentro do parêntese é negativo, a associação é inversamente proporcional, enquanto que, se o valor for positivo, a associação é diretamente proporcional. Dessa maneira, os maiores valores (mais positivos) na variável antropométrica da "média da dobra subescapular", ocorreram nas associações com os domínios físico, psicológico e social; já pelo contrário, os menores valores (mais negativos) se deram entre a associação da variável atlética "salto com auxilio" e os domínios físico, psicológico, social, na mesma modalidade. 
Focando no âmbito individual, as variáveis que possuem valores mais altos (tanto positivamente, quanto negativamente), na modalidade aquática, foram: flexibilidade, associada ao domínio físico; salto sem auxílio e $\mathrm{VO}^{2}$, com o psicológico; IMC e média da dobra supra-ilíaca, com o social; média da dobra supra-ilíaca, flexibilidade e freqüência cardíaca, com o ambiental.

\section{DISCUSSÃO}

Dada a amplitude das informações apresentadas de natureza estritamente atlética e sua correspondente interpretação corrente, interessa focar os resultados obtidos referentes à relação das mesmas com Qualidade de Vida (QV). Esta transita e transcende os aspectos antropométricos e atléticos tomados isoladamente, pois segundo o WHOQOL Group ${ }^{(16)}$ trata-se de "a percepção do indivíduo de sua posição na vida, no contexto da cultura e sistema de valores nos quais ele vive e em relação aos seus objetivos, expectativas, padrões e preocupações". Minayo(17) situa que é "uma noção eminentemente humana que tem sido aproximada ao grau de satisfação encontrado na vida familiar, amorosa, social e ambiental, e à própria estética existencial". Ainda é necessário considerar a conciliação entre condições de vida, como o local de moradia, meio de transporte, saneamento básico, entre outros, e estilo de vida, produto da subjetividade individual a modelar o sentido de tais objetividades ${ }^{(18)}$.

Interagindo com o antropométrico e o atlético, de fato, autores apontam que o treinamento regular e o grau de independência e mobilidade trazido pela vida ativa estão estritamente ligados à qualidade de vida da pessoa ${ }^{(19,20)}$. Nesse sentido, apesar de iniciarem a prática sistematizada, no nível classificado como sedentárias, após 16 semanas constatou-se diferença estatisticamente significativa apenas no domínio físico do treinamento em água. As que treinaram em água apresentaram mais resultados favoráveis nas variáveis de medidas corporais e atléticas do que as que faziam em solo, o que possivelmente teria repercutido na resposta dada a esta questão, de natureza subjetiva que é.

Tomando qualidade de vida e atividades aquáticas entre nós, estes resultados contradizem os de Vicentin et al.(21), em programa de hidroginástica com 40 mulheres, inicialmente sedentárias, igualmente jovens, que registraram diferenças não só no referente ao físico, mas igualmente no psicológico e meio-ambiente após 13 semanas de treinamento. Também no meio líquido, Pasetti et al. ${ }^{(22)}$ obtiveram bons resultados em obesas, no caso com o "deep water running", na melhora da percepção de QV em três (físico, psicológico e relações sociais) dos quatro domínios analisados, sugerindo que exercícios possam influenciar nestes aspectos. No exterior, Penedo et al. ${ }^{(23)}$, em revisão bibliográfica, também sugerem que o treinamento é benéfico física e mentalmente.

Entende-se, no entanto, que, na impossibilidade de adotar-se a prática do mini-trampolim na água, sua aplicação, em solo não deva ser desconsiderada, pois aí também diferenças significativas foram registradas nesta oportunidade na comparação de valores antes e após intervenção. De fato, Fontaine et al. ${ }^{(24)}$, medindo qualidade de vida com questionário SF-36, encontraram diferenças sobre o domínio físico, em homens e mulheres em sobrepeso, pela influência de treze semanas de treinamento. Ueda(25), em estudo com mulheres, após doze semanas de treinamento aeróbio de baixa intensidade, 3 vezes semanais, registrou efeitos favoráveis sobre o estado mental das voluntárias avaliadas por questões desenvolvidas por Sugiyama (1996), a despeito de Ransdell et al. (2005), lidando com três gerações de mulheres da mesma família e a influência de seis meses de exercícios, não terem detectado incremento nos escores sobre percepção de satisfação individual.

\section{CONCLUSÃO}

Considerando a impossibilidade de generalização dos achados fase a respectivas limitações e características, como o período adotado de dezesseis 
semanas de aplicação e avaliação, o significado desta investigação aponta, em termos de possíveis usos generalizados, que a modalidade aquática do mini-trampolim mostrou resultados mais expressivos do que o terrestre no referente ao domínio físico da Qualidade de Vida, medido pelo WHOQOL-Bref e variáveis antropométricas e atléticas. Ainda assim, as diferenças significativas verificadas na comparação entre os valores antes e após intervenção igualmente recomendam sua adoção, sobretudo em situações em que aquela não seja viável.

\section{REFERÊNCIAS BIBLIOGRÁFICAS}

1. Powers SK, Howley ET. Fisiologia do exercício: teoria e aplicação ao condicionamento e desempenho. São Paulo: Manole, 2000.

2. Hardman AE. Physical activity and health: current issues and research needs. Int J Epidemiol 2001;30:1193-7.

3. Mensink GBM, Loose N, Oomen CM. Physical activity and its association with other lifestyle factors. Eur $\mathrm{J}$ Epidemiol 1997;13:771-8.

4. ACSM. Programa de Condicionamento Físico do American College of Sport and Medicine. São Paulo: Manole, 1999.

5. ACSM. Position Stand on Progression Models in Resistance Training for Healthy Adults. Med Sci Sports Exerc 2002;34(2):368-80.

6. Oliveira Filho A, Shiromoto RN. Efeitos do Exercício Físico Regular sobre Índices Preditores de Gordura Corporal: Índices de Massa Corporal, Relação CinturaQuadril e Dobras Cutâneas. Rev Bras Educ Fis 2001;12(2):105-12.

7. Furtado E, Simão R, Lemos A. Análise do consumo de oxigênio, freqüência cardíaca e dispêndio energético, durante as aulas do Jump Fit. Rev Bras Med Esporte 2004;10(5):371-5.

8. Campbell DT, Stanley JC. Delineamentos experimentais e quase-experimentais de pesquisa. São Paulo: Editora Pedagógica e Universitária, 1979.

9. Heyward VH, Stolarczyk LM. Avaliação da Composição Corporal Aplicada. São Paulo: Manole, 2000.
10. Gordon CC, Chumlea WC, Roche AF. Stature, recumbent length, weight. In: Lohman TG, et al, editors. Anthropometric Standardizing Reference Manual. Champaign, Illinois: Human Kinetics Books, 1988.

11. Harrison GG, Buskirk ER, Lindsay Carter JE, Johnston FE, Lohman TG, Pollock ML, Roche AF, Wilmore JH. Skinfold thicknesses and measurement technique. In: Lohman TG, Roche AF, Martorell R, editors. Anthropometric Standardization Reference Manual. Champaign: Human Kinetics 1988.

12. Fleck MPA, Louzada S, Xavier M, Chachamovich E, Vieira G, Santos L, Pinzon V. Aplicação da versão em português do instrumento abreviado de avaliação da qualidade de vida WHOQOL-Bref. Rev Saúde Públic 2000;34(2):178-183.

13. Norman GR, Streiner DL. Bioestatistics- The base essentials. St. Louis: Mosby year book, 1994.

14. Johnson RA, Wicher NDW. Applied multivariate statistical analysis. $5^{\text {th }}$ ed. New Jersey: Prentice-Hall, 2002.

15. Gonçalves A. Os testes de hipóteses como instrumental de validação da interpretação (estatística inferencial). In: Marcondes MA, Lakatos CM, editores. Técnicas de pesquisa. São Paulo: Atlas, 2007;218-26.

16. The Whoqol Group. The World health organization quality of life assessment: position paper from the world health organization. Soc Sci Med 1995; 41:1403-9.

17. Minayo MCS. Qualidade de vida e saúde: um debate necessário. Ciênc Saúde Coletiva 2000;5(2):7-18.

18. Gonçalves A. Apresentação In: Gonçalves A, Vilarta R. Qualidade de vida e atividade física: explorando teorias e práticas. São Paulo: Manole, 2004; XIII.

19. Sparling PB. Are Wellness/Fitness programs benefiting participants' movement and mobility in daily life? Quest 2005;57:162-70.

20. Rikli RE. Movement and mobility influence on successfull aging: addressing the issue of low physical activity. Quest 2005;57:46-66.

21. Vicentin APM, Gonçalves A, Padovani CR, Aragon FF. Efeitos de programa quasi-experimental de hidroginástica na qualidade de vida de mulheres sedentárias aponta para consideração do domínio social no WHOQOL-Bref. In: ॥ Encontro Ibero-Americano de Qualidade de Vida. Porto Alegre, RS: 19-21 de agosto de 2004. 
22. Pasetti SR, Gonçalves A. Padovani C. Deep water running e melhora da qualidade de vida em obesas. In: II Encontro Ibero-Americano de Qualidade de Vida. Porto Alegre, RS: 19-21 de agosto de 2004.

23. Penedo F, Dahn LA, Jason R. Exercise and well-being: a review of mental and physical health benefits associated with physical activity. Curr Opin Psychiatry 2005; 18(2):18993.

24. Fontaine KR, Barofsky I, Andersen RE, Bartlett SJ, Wiersema LC. Impact of weight loss on health-related quality of life. [Abstract]. Qual Life Res 1999;8(3):275-7.

25. Ueda MA. 12-week structured education and exercise program improved climacteric symptoms in middleaged women. J Physiol Anthropol Appl Hum Sci 2004; 23:143-8.

26. Sugiyama M. Study on quality of life and nutrition defect start in the menopause. Grants-in-Aid for Scientific Research Report, 1996;19-22.
27. Ransdell LB, Robertson L, Ornes L, Moyer-Mileur L. Generations Exercising Together to Improve Fitness (GET FIT): A Pilot Study Designed to Increase Physical Activity and Improve Health-Related Fitness in Three Generations of Women. Women \& Health 2005;40(3):79-96.

Aguinaldo Gonçalves - Médico Sanitarista Professor Titular, FEF/UNICAMP aguinaldo@fef.unicamp.br , aguinaldogon@uol.com.br http://www.unicamp.br/fef/grupos/gsceaf/index.htm Rua Luverci Pereira de Souza, 1151 Cidade Universitária - Barão Geraldo 13 083-730 Campinas-SP

Fone 19-32895022 